\title{
Experimental Measurements and Antenna Isolation for TETRA Communication System in Underground Mining and decline
}

\author{
Batzorig Bazargur', Otgonbayar Bataa' ${ }^{1}$, Zagarzusem Khurelbaatar1, Batbayar Battseren ${ }^{1}$ \\ 1 School of Information and Communication Technology, \\ Mongolian University of Science and Technology \\ batzorigbazargur@gmail.com, otgonbayar b@must.edu.mn, zagarzusem@must.edu.mn, \\ nano.batbayar@gmail.com
}

\begin{abstract}
:
Mining industries play an important role in the economic development of Mongolia. In mining operations, truly reliable and robust communication systems play a vital role in ensuring personnel safety, enhancing operational efficiency and process optimization. In this thesis, we have carried out the calculation of RF amplifier wave propagation in mines and in special-purpose tunnels, in tunnel entrance and in box cut declines of tunnel entrance. UHF radio frequency radiation tests have been carried out in the 16-degree box cut declines at a depth of 30 meters and in the 150m long tunnels beneath it. In this case, it is necessary to create significant isolation between the donor antenna and the service antenna of RF amplifier. Our results are useful to understand the isolation of antennas and implications of the physical environment, signal radiation characteristics in underground mine communication system.
\end{abstract}

Key words: tunnel, propagation calculation, UHF frequency, RF amplifier, decline.

\section{Introduction}

We need to provide reliable radio communication services in tunnels for different purposes and in the decline (underground mines, subway tunnels etc.) or in smalldiameter, deep holes where the radio communication signal strength is so weak. Installing fiber optic amplifier or a base station is highly reliable and easy to install in such environments, but it leads to prohibitive cost and time. There is no ready to use model for calculating and modelling radio wave propagation as well. So, radio wave propagation modeling plays a vital role to solve such problems which is possible with RF amplifier. RF amplifier model utilized in underground mines is shown in Figure 2.

The Scope of works is for the provision of civil construction and tunneling services for the associated portal structures, twin parallel declines and conveyor transfer and drive station excavations to facilitate installation of ore handling system and service access to underground shaft mine, and associated services.

\section{- $\quad 6.6 \mathrm{~km}$ long twin parallel tunnel}

\section{- $\quad 16.6 \mathrm{~km}$ tunneling}

The decline is a dual heading system with parallel declines each consisting of three legs required for conveyors and mine access. chambers

$16.6 \mathrm{~km}$ tunnels \& 34,000 cu.m large

- $\quad$ Leg length approx. 2200m

- $\quad$ Final depth $1120 \mathrm{~m}$

- Gradient max $18.5 \%$

- $\quad$ Crosscut every $200 \mathrm{~m}$

- $\quad$ Safety bay every $30 \mathrm{~m}$

10.2 angle is the maximum grade range of decline which can provide safest working condition in order to reach $1300 \mathrm{~m}$ deep.

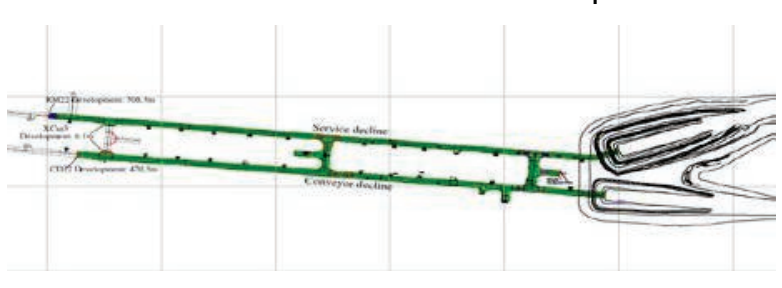

Fig. 1. Decline and tunnel 
RF amplifier is used in order to meet the radio communication needs during the Phase 1 construction work means early stage of this project (150-200m tunnel). It is comprised of the following main components such as donor of amplifier, service antenna, isolation and amplifier. RF amplifier is developed to provide radio communication network in the decline of conveyor transfer and in the decline of the first 150-200m depth.

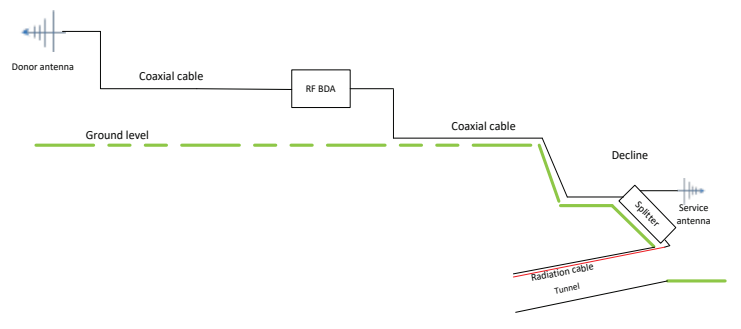

Fig. 2. Geometric modelling of RF amplifier connection diagram for decline and tunnel

\section{Background}

In this thesis, we have carried out the calculation of RF amplifier wave propagation in tunnels, radiating cable modelling and wave propagation modelling in tunnel entrance and in the declines. In order to provide effective construction activity as well as safety control, executive should have reliable radio communication coverage around the decline construction area. Before two-way radio coverage couldn't cover under the surface level and decline tunnel therefore additional radio coverage expansion requires around the construction area. Radio coverage will be expanded by step by step to align with construction activity:

\section{Phase 1.}

\section{- $\quad$ To cover decline area}

- $\quad$ To cover extended range (100-150m) of decline tunnel construction. RF amplifier is used as a temporary solution at a depth of 150-200 meters in the decline. RF amplifier test has not been carried out in longer tunnels. Permanent solution to this is DAS based solution connected to BTS. We have used RF amplifier, donor and service antenna (both are Yagi antenna), coaxial and leaky radiation cables for the temporary solution.

In [1], repeater amplifiers, also known as signal boosters, are specialized RF systems that extend radio coverage into enclosed or shadowed areas where abrupt propagation losses impair communication.

Materials such as soil or rock, brick, cement, reinforced concrete, metals, and metal-coated thermal glass panes are notorious for their ability to block electromagnetic radiation in the radio frequency range. In the interior of structures made of those materials, and in areas where natural or man-made structures block radio propagation, radio frequency levels may be 30 to $100 \mathrm{~dB}$ or more below unobstructed levels (nothing but cosmic ray particles and neutrinos penetrates into deep mines, for example). Repeater amplifiers boost radio signals to levels sufficiently high to provide reliable communication in those enclosed or blocked areas.

Repeater amplifiers have acquired great prominence in the radio communication industry in the last few years, due to a rapidly growing demand for extended communications services inside all types of urban structures. However, they made their first appearances several decades ago, as a part of "leaky feeder" or "leaky coax" radio communication systems in underground mines, vehicular and railroad tunnels. One-way repeater amplifiers were used in various configurations for simplex and semiduplex radio communication in underground tunnels. In another paper, the authors looked the tunnel propagation model, and in this paper a fully vector finite element base propagation model is developed for blocked straight and curved tunnels. Effect of different vehicles, location of vehicles and number of vehicles inside tunnels is analyzed [2]. Some researcher studied to look at the frequency band techniques in Indian underground mining case. The paper discusses different radio frequency communication techniques being employed for Indian underground mines. Experiments were conducted in the laboratory as well as in the underground coal mines for medium wave frequency (MF), very high frequency (VHF) and ultra-high frequency (UHF) electromagnetic propagation as well as induction technique to meet different types of mining conditions [3]. On the other hand, they looked the trends of the tunnel propagation model. The authors developed current and future trends in technology, applications and propagation modeling are also identified. About ninety relevant references have been reviewed that consider: 1) the emergence of technology and applications, 2) analytical, numerical and measurement based propagation modeling techniques, and 3) implications of the physical environment, antenna placement and radiation characteristics on wireless communication system design. Affected systems include narrowband, wideband/ultra-wideband (UWB) and multiple-antenna systems. The paper concludes by identifying open areas of research [4]. To put it another way, above researchers have presents that results showing the 
accuracy of the ADI technique when used to model the parabolic equation for (a) square (b) circular and (c) semi-circular cylindrical PEC tunnels. For each tunnel, we compare the numerical solution with the known analytical solution for different discretization along the transverse plane and propagation axis [5]. They are also maintains that explores extend the analysis of this method by including the realistic cases of branching tunnels and tunnels with rough walls [6]. The paper [7], concludes that the ADI-PE, shows simulation results for tunnel test cases with known analytical solutions. The author propose that configure a radio transceiver, designers must understand the performance parameters and tradeoffs of the RF amplifiers being used. A multitude of system requirements must be considered to choose an optimal amplifier from the many devices available by suppliers in the marketplace [8]. The paper notes that focuses on the modern linearizing techniques for the high power transmit amplifiers and reports on efforts to evolve system and behavioral level performance measures and quantifying techniques which will aid processes such as the design, specification and evaluation of linearizers and their overall contribution to transmit channel performance [9]. In [10] this doctoral thesis has focused on Doherty amplifiers (dynamic load technique) and high efficiency class-E amplifiers (main amplifier in EER) applied to WiMAX at $3.5 \mathrm{GHz}$. This paper investigation that the amplifier requirements, the proposed solutions and their status [11]. All cavities work at $88 \mathrm{MHz}$, are independently phased and powered by amplifiers whose power ranges from few kilowatts to $250 \mathrm{~kW}$. Research in tunnel wireless communication [12], the author describes the various assumptions used in the design and analysis of distributed antenna system (DAS) for trains, tunnels and in-building wireless radio coverage. The design includes handover overlap design, base station connectivity, signal reticulation using splitters, couplers, bidirectional amplifiers, attenuators, discrete antennas, radiating cables and optic-electric couplers etc.

\section{Radio Frequency (RF) Isolation}

In this section, we focus our attention on the RF isolation between donor and service antenna. Obtaining isolation between donor antenna and service antenna is one of the most important factors to provide radio network in tunnels and decline using RF amplifier. However, it provides acceptable results when such tunnels and declines are located close to the base station (Receiver antenna gain and a donor antenna gain of RF amplifier can be $-75 \mathrm{~dB}$ and more). Antenna isolation is a key consideration in the design of any radio communications system. Sufficient isolation is required to ensure that interference between systems is kept within acceptable levels (levels at which the equipment can operate effectively).

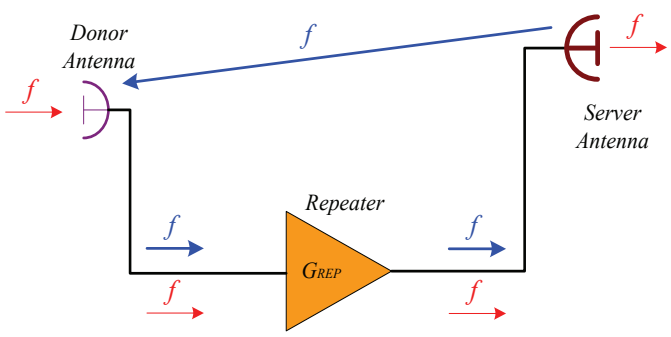

Fig. 3. Radio frequency (RF) amplifier isolation.

To let repeater works well, F>G+15Db.

$\mathrm{F}$ : Isolation between the Donor antenna and service antenna.

G: Gain of the repeater

$I_{h}=22.0+20 \log 10(d / \lambda)-\left(G_{d}+G_{r}\right)+\left(X_{d}+X_{r}\right)+C$

$G_{d}, G_{r}$ is the gain of donor and server

$X_{d}, X_{r}$ is the Front to Back ration for antennas.

In Oyu Tolgoi mine, we suppose $\mathrm{f}=400 \mathrm{MHz}$, $\lambda=0.75 \mathrm{~m}$.

Suppose $\mathrm{G}_{\mathrm{d}}=10 \mathrm{~dB}, \mathrm{X}_{\mathrm{d}}=20 \mathrm{~dB}, \mathrm{G}_{\mathrm{r}}=10 \mathrm{~dB}, \mathrm{X}_{\mathrm{r}}=$ $16 \mathrm{~dB}$. $C$ is the loss by obstacles. Suppose $C=0$ here.

Tab. 1: Radio frequency (RF) horizontal isolation

\begin{tabular}{|c|c|c|c|c|c|c|}
\hline $\mathrm{D}$ (distance) & 3 & 5 & 8 & 10 & 15 & 20 \\
\hline horizontal isolation(dB) & 50 & 54 & 59 & 60 & 64 & 67 \\
\hline maximum gain(dB) & 35 & 39 & 44 & 45 & 49 & 52 \\
\hline
\end{tabular}

The calculator provides an estimate of the horizontal and vertical isolation provided by two $2-3 m$ separated antennas. The spacing between a donor antenna and a service antenna is 3$58 \mathrm{M}$.

$I_{v}=28.0+40 \log 10(d / \lambda)+C$

$\mathrm{C}$ is the loss by obstacles. Suppose $\mathrm{C}=0$ here In OT, suppose $\mathrm{f}=400 \mathrm{MHz}, \lambda=0.75 \mathrm{~m}$

Tab. 1: Radio frequency (RF) vertical isolation results

\begin{tabular}{|c|c|c|c|c|c|c|}
\hline $\mathrm{D}$ (distance) & 3 & 5 & 8 & 10 & 15 & 20 \\
\hline vertical isolation(dB) & 52 & 61 & 69 & 73 & 80 & 85 \\
\hline maximum gain(dB) & 37 & 45 & 54 & 57 & 65 & 70 \\
\hline
\end{tabular}




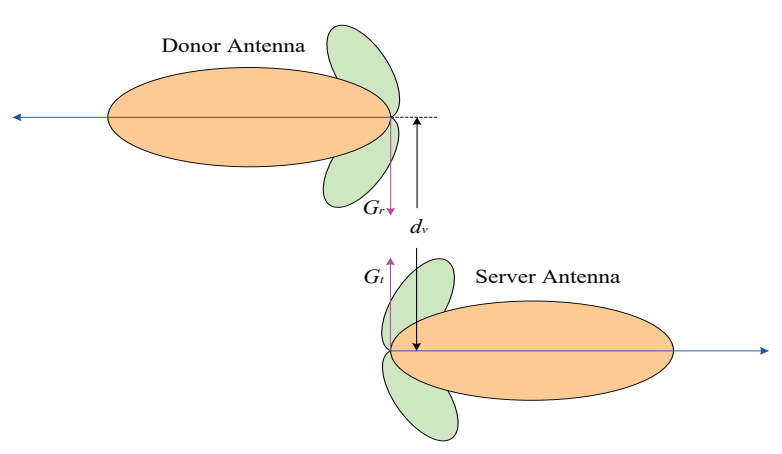

Fig. 4. Radio frequency (RF) vertical isolation

With same distance, vertical isolation is larger than horizontal isolation. So it is better to use vertical isolation [13].

\section{Modelling of radio wave propagation in tunnels}

Radiating cables are widely used to ensure radio communication in tunnels, but they have no amplifier like antennas. Attenuation coefficient of radiating cables is different from antenna's attenuation coefficient. There are two main types of losses: Longitudinal loss and transmission loss. Transmission loss has 2 different measurements: C95 (95\% percentile of the coupling loss) and C50 (median value of the coupling loss). These are predicted measurements of received signals via radiating cables [13].

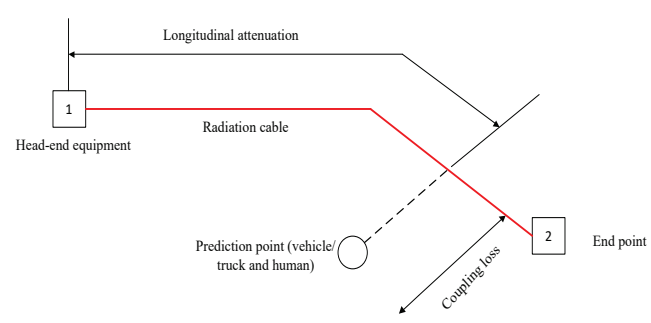

Fig. 5. Geometric modelling of radiating cables propagation

\section{Calculating of RF amplifier wave propagation in tunnels}

The RF amplifier radio communication network in tunnel entrance is provided by the service antenna isolation under the decline and in tunnels, radio communication network is enabled by radiating cables via two-way splitter. The calculation of this wave propagation modelling is carried out as follows:

$$
P=P i-L 1-L 2
$$

For leaky cable, suppose cable was install tunnel side wall, and following:

- Suppose $P$ is the signal power at a tunnel point 2 meters away from leaky cable.
- $\mathrm{Pi}$ is the RF power input the leaky cable

- $\quad$ L1 is the cable transmission loss from cable input point to the point of testing location ( $P$ point)

- $\quad$ L2 is the leaky cable coupling loss (95\% coupling loss)

If mobile locate far from 2 meters, then additional small loss will have, but if the mobile is inside a vehicle, then about additional more $15 \mathrm{~dB}$ loss must be considered on $800 \mathrm{MHz}$ (for our case we supposed the $17 \mathrm{~dB}$ loss considered on our TETRA band $400 \mathrm{~Hz}$ ) [14].

We used RLK78-50JFNA model radiating cable in this calculation. Following that, we have carried out the line and cable calculation in tunnels. Based on the results of a calculation, we have determined how many meters of radiating cables are needed in tunnel.

Link Budget $=T X$ power + abs (Rx sensitivity) (all additional attenuations) - Coupling loss

Cable Length $=$ Link Budget $/$ Longitudinal Loss $(d B / 100 m) * 100$

\section{Calculation of radio wave propagation modelling in the decline of tunnels}

Okumura-Hata model is used to calculate wave propagation in the tunnel entrance as it is shown in (see Fig. 2).

$L_{u}=69.55+26.16 \log _{10} f-13.82 \log _{10} h_{b}-C_{H}+[44.9-$

$\left.6.55 \log _{10} h_{b}\right] \log _{10} d$

- Lu-is the path loss $(\mathrm{dB})$

- $h_{b}$-is the base station antenna height (in meters)

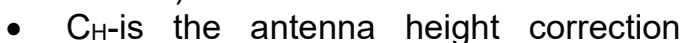
factor (dependent of environment, frequency and mobile height)

- $\quad \mathrm{f}$-is the transmission frequency $(\mathrm{MHz})$

- $d$-is the distance between transmitter and receiver (in kilometers).

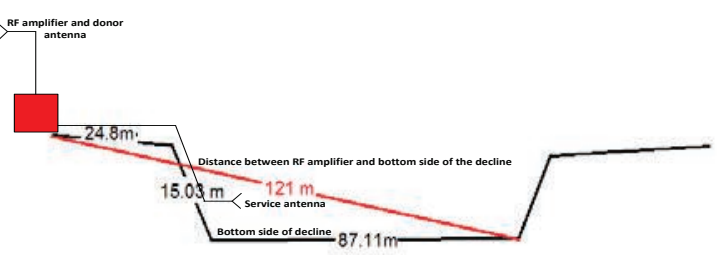

Fig. 6. Geometric modelling of decline flat area

We used service Yagi antenna specification, the antenna horizontal beam width is 65 degree, vertical beam width is 105 degree, and gain is $6 \mathrm{dBd} / 8 \mathrm{dBi}$. The area service antenna covered could be regarded as a flat area, so, free space propagation formula could be used. Assuming antenna height is 15.03 meters, repeater output power is $43 \mathrm{dBm}$. 
Comparative data of $400 \mathrm{MHz}$ and $800 \mathrm{MHz}$ frequencies of RF amplifier isolation and signal power in tunnels is illustrated in Figure 7 and Figure 8 respectively.

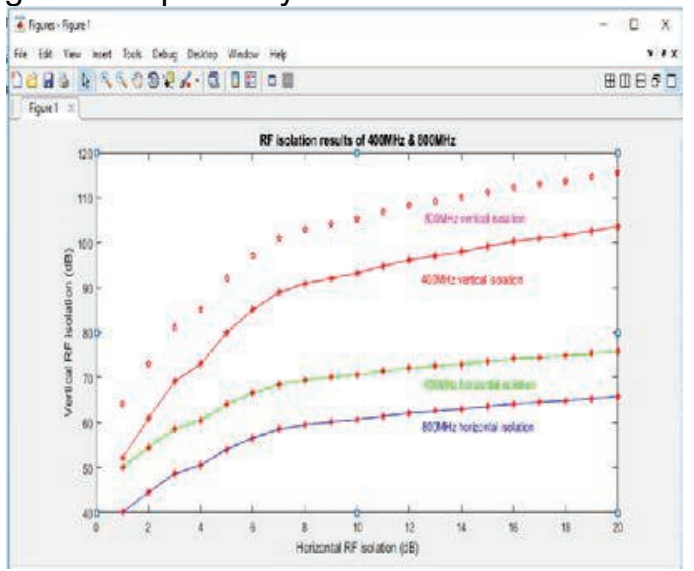

Fig. 7. Horizontal and vertical RF isolation results both on $400 \mathrm{MHz} \& 800 \mathrm{MHz}$

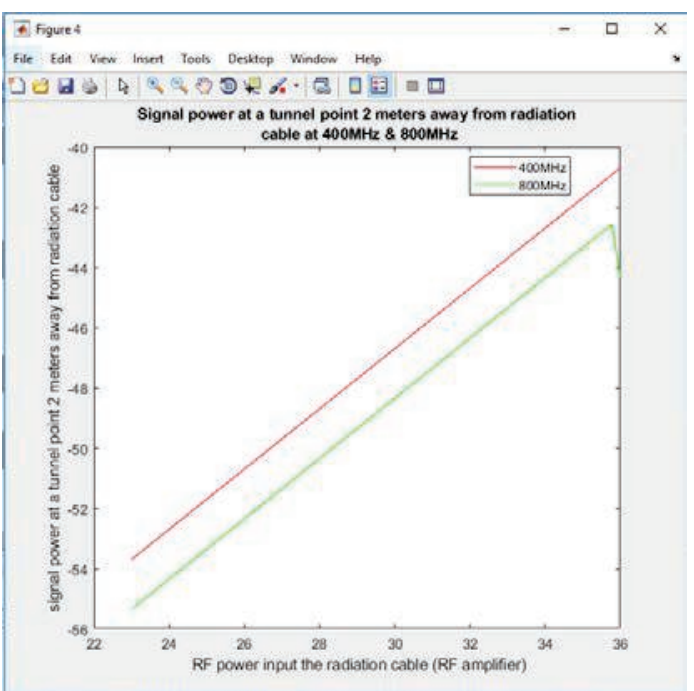

Fig. 8. Horizontal Signal power at a tunnel point 2 meter away from radiation cable at $400 \mathrm{MHz} \&$ $800 \mathrm{MHz}$

This was successfully tested and deployed in the decline of Oyu Tolgoi mining in 2016.

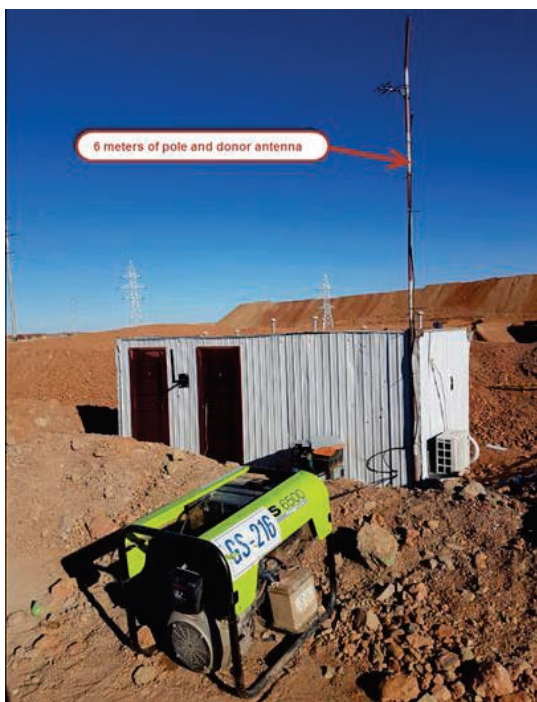

Fig. 9. RF donor antenna location

In order to provide radio communication network below the decline of tunnel entrance, we used a service antenna isolation with metal board.

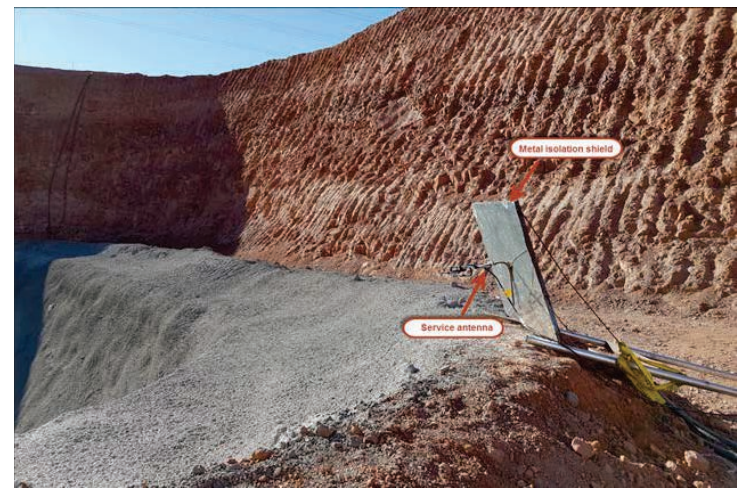

Fig. 10. RF metal isolation between donor and service antenna

In tunnels, we used radiating cables fixed on the wall of a tunnel and Yagi antenna was utilized at the end of radiating cables.

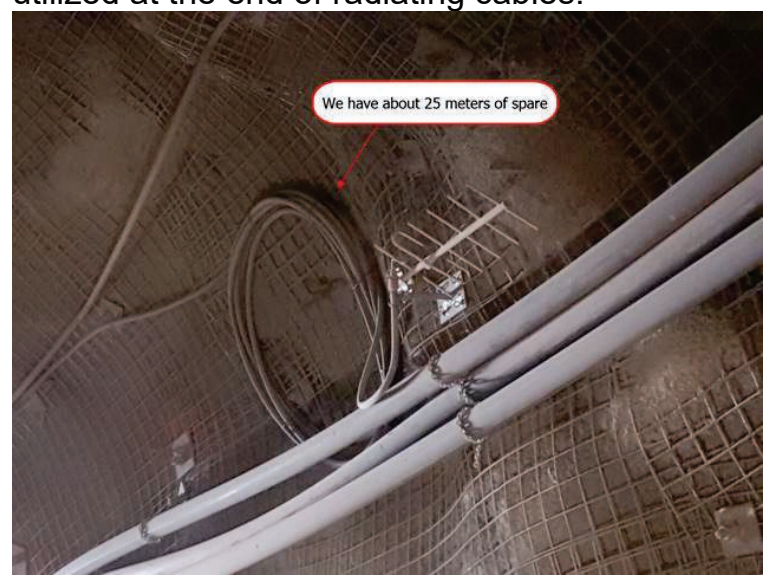

Fig. 11. End point of the radiation cable 


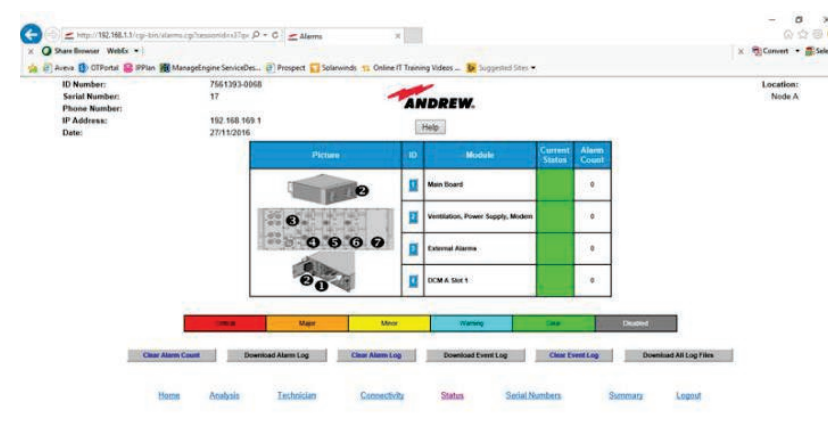

Fig. 12. RF amplifier's normal status

After utilizing RF amplifier, signal strength received by the user TETRA handheld radio terminal has been improved from $-89 \mathrm{~dB}$ to $64 \mathrm{~dB}$. This outcome is shown in Figure 13.

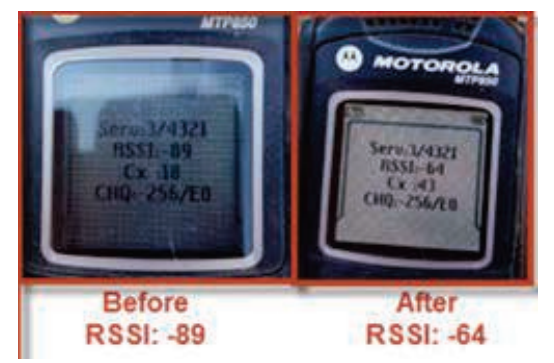

Fig. 13. TETRA handheld radio's RSSI results before and after

\section{ConClusion}

In this thesis, the calculation of wave propagation has been carried out to utilize RF amplifier in tunnels. One of the most important thing is to provide isolation between a donor antenna and a service antenna of RF amplifier. Because the better the isolation is provided, the higher antenna gain there will be on a service antenna. Particularly, it would be more effective when we provide horizontal isolation. It is clearly seen from Figure 5 and Table 1.

Besides that, we have carried out calculation of tunnel wave propagation based on RF amplifier. In order to provide better isolation, we can increase spacing between a donor antenna and a service antenna, use metal wall or large buildings if possible. In case if a service antenna is not utilized, it would be easier to calculate wave propagation in tunnels using RF amplifier and to provide radio communication network. Because the spacing between a tunnel and a donor antenna would be a good isolation. RF amplifier enables radio communication network in the declines and tunnels. It provides low cost and more reliable services compared to base stations and optical amplifier. Further we need careful and accurate calculation of influence of obstacles on wave propagation in tunnels. It plays an important role in predicting radio wave propagation in tunnels.

\section{References}

[1] Ernesto A. Alcivar, Repeater Amplifier Systems: Principles and Applications, 1994 TX RX Systems Inc. p.1.

[2] Kamran Arshad, Ferdinand Katsriku, Aboubaker Lasebae, MODELLING OBSTRUCTIONS IN STRAIGHT AND CURVED RECTANGULAR TUNNELS BY FINITE ELEMENT APPROACH, Journal of ELECTRICAL ENGINEERING, VOL. 59., NO. 1, 2008, p.9.

[3] L. K. Bandyopadhyay, P. K. Mishra, Sudhir Kumar and A. Narayan, RADIO FREQUENCY COMMUNICATION SYSTEMS IN UNDERGROUND MINES", p.1.

[4] Arghavan Emami Forooshani, Shahzad Bashir, David G. Michelson, Senior Member, IEEE, and Sima Noghanian, Senior Member, IEEE, A Survey of Wireless Communications and Propagation Modeling in Underground Mines, 1553-877X/13/\$31.00 @ 2013 IEEE, p.1.

[5] Richard Martelly and Ramakrishna Janaswamy, Propagation in Tunnels Using the Parabolic Equation and the ADI Technique, 1-4244-08784/07/\$20.00 @2007 IEEE, p.45.

[6] R. Martelly, R. Janaswamy, Propagation Prediction in Rough and Branched Tunnels by the ADI-PE Technique, 978-1-4244-33865/09/\$25.00 @2009 IEEE, p.596.

[7] Richard Martelly and Ramakrishna Janaswamy, Fellow, IEEE, An ADI-PE Approach for Modeling Radio Transmission Loss in Tunnels, 0018926X/\$25.00 @ 2009 IEEE, p.1759.

[8] Tuan Nguyen, Choosing the Proper RF Amplifier Based on System Requirements, WJ Communications, Inc., San Jose, CA, p.1

[9] M. O'Droma, N. Mgebrishvili, A. Goacher, LINEARITY AND EFFICIENCY ISSUES IN RF POWER AMPLIFIERS FOR FUTURE BROADBAND WIRELESS ACCESS SYSTEMS, ECE Dep., University of Limerick, Ireland, p.1.

[10] Manuel Yarlequé, RF POWER AMPLIFIERS FOR WIRELESS COMMUNICATIONS, $P h D$ thesis, June 2008, p.v.

[11] Marco Di Giacomo, Bernard Ducoudret, RF POWER AMPLIFIERS FOR THE SPIRAL 2 DRIVER: REQUIREMENTS AND STATUS, Proceedings of LINAC08, Victoria, BC, Canada, p.897.

[12] S. K. Palit, DESIGN OF WIRELESS COMMUNICATION SENSING NETWORKS FOR TUNNELS, TRAINS AND BUILDINGS, INTERNATIONAL JOURNAL ON SMART SENSING AND INTELLIGENT SYSTEMS, VOL. 2, NO. 1, MARCH 2009, p.118.

[13] Telco Authority, Antenna Placement and Isolation Guideline, March 2015, p.4 and p.14.

[14] Simon R. Saunders, Alejandro Aragon-Zavala, Antennas and Propagation for Wireless 
Communication Systems: 2nd Edition, (May 7, 2007).pp.315-317 